\title{
Plan de reducción de pérdidas de energía de la Corporación Nacional de Electricidad Regional Santo Domingo (CNEL), en la provincia de Santo Domingo de los Tsáchilas.
}

\section{Plan for the reduction of energy losses of the National Corporation for Regional Electricity Santo Domingo (CNEL), in the province of Santo Domingo de los Tsáchilas.}

Jorge Luis Puyol Cortez ${ }^{1}$,Evelyn Karina Tinoco Diaz. ${ }^{2}$, Verónica Janneth Castro Celi, ${ }^{3}$, Evelyn Eugenia Alcívar Soria ${ }^{4} \&$ Efraín Velasteguí

\section{DOI: https://doi.org/10.33262/visionariodigital.v3i1.130}

\section{Resumen.}

Este trabajo de investigación se enfoca, en el plan de reducción de pérdidas de energía de la Corporación Nacional de Electricidad CNEL, de la provincia de Santo Domingo de los Tsáchilas promoviendo una campaña de concientización ciudadana para evitar el hurto de este servicio.

Durante el periodo de abril (Domingo, 2012) se indagó en los sectores de los barrios bajos de Santo Domingo el problema el cual tiene la otra cara de la moneda, pues existen

\footnotetext{
${ }^{1}$ Universidad Técnica Luis Vargas Torres, Facultad de Ciencias Administrativas, Santo Domingo, Ecuador, jpuyol73@gmail.com

${ }^{2}$ Universidad Técnica Luis Vargas Torres, Facultad de Ciencias Administrativas, Santo Domingo, Ecuador, kaevecris@hotmail.com

${ }^{3}$ Universidad Técnica Luis Vargas Torres, Facultad de Ciencias Administrativas, Santo Domingo, Ecuador, verito952012@hotmail.com

${ }^{4}$ Universidad Técnica Luis Vargas Torres, Facultad de Ciencias Administrativas, Santo Domingo, Ecuador, evelyn_alcivar@hotmail.com
} 
innumerables asentamientos que carecen de servicios básicos como el de la energía eléctrica. En estas zonas los abonados se conectan directamente de la red para tomar energía eléctrica, sin que este problema existente desde hace años haya podido ser atenuado con eficiencia y difícilmente podrían ser eliminadas por completo.

Así se detecta un problema de incremento de las pérdidas de energía en la corporación Nacional de electricidad CNEL Santo Domingo debido a la falta de equipos de medición, equipos obsoletos o en mal estado, así como redes desnudas que dan pasó a las conexiones directas.

El plan propuesto para la recuperación de las pérdidas no técnicas del sistema CNEL Santo Domingo estima reducir las pérdidas en un lapso no superior a un año, para así lograr incrementar el flujo de caja que proporcione sustentabilidad financiera y permita el pago de los valores facturados por generadores y transmisor, así como lograr su objetivo principal: Disminuir las pérdidas que se han venido dando durante años, para lo cual se deberá emplear personal técnico capacitado y los materiales que cumplan con las normas de calidad especificadas.

Por tal virtud se recomienda una actualización permanente de su base de datos, con el fin de disponer de información precisa y confiable para el análisis de pérdida en el sistema de comercialización, para de esta forma concientizar al cliente con el fin de optimizar el uso de energía eléctrica y con esto eliminar el fraude mediante la aplicación de todos los mecanismos necesarios.

Palabras claves: plan de reducción de pérdida de energía CNEL, redes desnudas

\section{Abstract.}

This research work focuses on the energy loss reduction plan of the National Electricity Corporation CNEL, in the province of Santo Domingo de los Tsáchilas, promoting a public awareness campaign to prevent the theft of this service.

During the period of April (Sunday, 2012) the problem was questioned in the neighborhoods of Santo Domingo, which has the other side of the coin, since there are innumerable settlements that lack basic services such as electricity. In these areas the subscribers are connected directly from the grid to take electric power, without this problem existing for years could have been attenuated with efficiency and could hardly be eliminated completely.

Thus, a problem of increased energy losses in the CNEL Santo Domingo National Electricity Corporation is detected due to the lack of measuring equipment, obsolete equipment or in poor condition, as well as naked networks that give way to direct connections. 
The proposed plan for the recovery of non-technical losses of the CNEL Santo Domingo system estimates to reduce losses in a period of no more than a year, in order to increase the cash flow that provides financial sustainability and allows the payment of the values billed by generators and transmitter, as well as achieving its main objective: Reduce the losses that have been occurring for years, for which you must employ trained technical personnel and materials that meet the specified quality standards.

For this reason, a permanent update of its database is recommended, in order to have accurate and reliable information for the loss analysis in the marketing system, in order to make the client aware in order to optimize the use of electric power and with this eliminate fraud by applying all the necessary mechanisms.

Keywords: CNEL energy loss reduction plan, naked networks

\section{Introducción.}

La Corporación Nacional de Electricidad Regional Santo Domingo ha sido una de las instituciones que ha influido de manera directa en el desarrollo de la ciudad de santo Domingo de los colorados, se inició el 22 noviembre del año 1963 como una pequeña empresa, con el pasar del tiempo se convirtió en una de las principales Instituciones generadoras de fuentes de trabajo y desarrollo para nuestra querida ciudad.

Sin duda alguna la energía eléctrica es uno de los principales elementos indispensables para la vida del ser humano, la gran cantidad de electrodomésticos que facilitan nuestra vida funcionan en base a energía eléctrica, convirtiéndose en una necesidad en todos los estratos sociales (Publicaciones, 2012).

Sin embargo, la situación económica por la que atraviesan los países en el mundo ha generado un porcentaje de pobreza muy alto, ya que las fuentes de trabajo se han visto limitadas, lo cual influye mucho en la economía familiar, esta quizá ha sido una de las causas principales para que exista el hurto de energía por parte de los clientes. Las distribuidoras de energía se han visto en serios conflictos financieros debido a que existe un alto porcentaje de hurto de energía, y de pérdidas técnicas las mismas que se ocasionan por la naturaleza del trabajo al momento de su instalación. Con el presente trabajo quiero proponer procedimientos y estrategias que nos ayuden a obtener un informe minucioso y detallado de los sectores más vulnerables y riesgosos para emprender con la aplicación de soluciones a este gran problema.

Realmente las perdías de energía de las empresas distribuidoras, son la suma de las ineficiencias que a lo largo del tiempo se han producido y reflejan los errores en la aplicación de criterios y técnicas incluyendo su sistema operativo. La falta en el control de Ingeniería permite que el avance de un sistema produzca más pérdidas, debido a que no se utilizan las 
herramientas ni los procedimientos necesarios. Dentro de las pérdidas de energía puede haber una infinidad de causas, pero es importante determinar cuáles debemos atacar para de esta manera aplicar un sistema ideal y recomendado para la empresa Distribuidora. Esto hace necesario un estudio claro y especifico.

Es un tema indiscutible el mejoramiento del manejo de la energía en las empresas distribuidoras y se hace necesario analizar la reducción y control de pérdidas en cada uno de ellas, mediante estudios que permitan lograr una red donde las pérdidas técnicas es decir aquellas causadas por los diversos fenómenos físicos que se presentan en los componentes de un sistema de distribución, lleguen a un nivel aceptable.

Este proyecto sugiere medidas tendientes a la reducción de pérdidas y al control del hurto de energía mediante la aplicación de un diseño metodológico en el que se analice tanto las pérdidas técnicas como las no técnicas. (Hernandez \& Palafox, 2012)

La modernización del sector eléctrico en el país lleva a las Empresas Distribuidoras de energía eléctrica a buscar un desarrollo integral en todas sus áreas. De este modo podrán cubrir su déficit y a la vez atender futuras inversiones para cubrir el crecimiento de su demanda eléctrica. Un medio de generación de fondos internos, es el implementar alternativas que permitan enmarcar la facturación de su energía disponible en niveles aceptables. Esto será un determinante para la eficiencia técnica, administrativa y comercial de la Empresa. Uno de los parámetros que permite evaluar la eficiencia de una Empresa Eléctrica es el nivel de sus pérdidas. Ya que, del valor y composición de éstas, dependerá la necesidad de realizar inversiones en su sistema eléctrico.

\section{Paradigma investigativo.}

Para realizar este trabajo, me he basado en estudios realizados en la universidad de Guayaquil, en la Escuela Politécnica, las mismas que han presentado trabajos con la misma problemática referente a las empresas eléctricas, además el Ministerio de Energía y Minas también presenta propuestas al igual que las empresas eléctricas en nuestro país, que quizá por problemas económicos o administrativos no han tenido éxito.

El departamento de control de pérdidas de CNEL Regional Santo Domingo me ha proporcionado la información necesaria, para hacer un verdadero análisis el mismo que me permitirá exponer soluciones muy prácticas que nos llevaran a corregir este grave problema También la información proporcionada por el Ministerio de Electricidad y Recursos Renovables, quien hizo un cuadro comparativo desde el año 2007 en el que las perdidas registraban el 21,42\% el mismo que se ha logrado disminuir al 15,58 \% 
Según cifras del Consejo Nacional del Electricidad (Conelec), las pérdidas detalladas de mayo 2010 a abril de 2011 son de $6,7 \%$ en pérdidas no técnicas o conocidas como hurto de energía

El restante $8,9 \%$ corresponden a pérdidas técnicas que se producen en el proceso físico para que la electricidad llegue desde los puntos de generación hasta el usuario

El ministro aseguró que varias han sido las medidas que se han tomado para bajar el porcentaje de pérdidas y promover la eficiencia energética. Una de ellas ha sido instalar equipos de medición en hogares donde no había y cambiarlos en aquellos que no registren el consumo.

\section{Muestra poblacional}

Tabla 1. Muestra Poblacional.

\begin{tabular}{llll}
\hline $\mathrm{N}^{\circ}$ & Concepto & Contenido & $\%$ \\
1 & Directivos & 58 & $5 \%$ \\
2 & Usuario & 5000 & $95 \%$ \\
& & & $\mathbf{1 0 0 , 0 0}$ \\
& Total poblacion & $\mathbf{5 0 5 8}$ &
\end{tabular}

Fuente de información: Archivo del departamento control de pérdidas de energía de CNEL

\section{Matriz muestral}

Tabla2. Matriz muestral.

\begin{tabular}{llll}
\hline $\mathrm{N}^{\circ}$ & Concepto & Contenido & $\%$ \\
1 & Personal de cnel & 20,05 & $5 \%$ \\
2 & Usuarios & 380,95 & $95 \%$ \\
& & \\
& & & 100
\end{tabular}

Fuente de información: archivo del departamento control de pérdidas de energía de CNEL.

\section{Método analítico}

\section{Metodología}

El método que se utilizará será el método analítico, ya que estudiaremos el problema y este será dividido en cuatro fases las cuales serán analizadas una a una para poder aplicar las posibles soluciones que proponemos en el presente proyecto. 
Método sintético.

Porque elaborare las conclusiones y las recomendaciones que me permita resumir la presente investigación, hacer énfasis en los resultados.

\section{Metodo estadistico}

Porque se elaboran cuadros de resultados con porcentajes tendientes a la recuperación de la cartera vencida y se analizara el porcentaje de pérdidas que han sido superadas.

\section{Métodos teóricos.}

Analítico. - con este método se estudiará el problema y se analizará su impacto.

Sintético. - se realizará sobre la base del análisis permitiendo relacionar los elementos entre sí y vincular la situación problema con un todo.

Inductivo y deductivo. - estos métodos permitirán al autor conocer el contexto del problema motivo de la presente investigación, desde un enfoque particular para arribar a lo general, en ciertos temas y en otros, se abordaron las cuestiones desde una visión amplia y generalizada para terminar en lo particular o en lo específico de determinada situación.

\section{Métodos empíricos.}

Los métodos empíricos a utilizarse serán las encuestas individuales y estructuradas a las diferentes personas vinculadas, la observación estructurada y las encuestas a los directivos, trabajadores, empleados y usuarios, con el propósito de obtener información sobre el fenómeno observado y confirmar la hipótesis.

\section{Método matemático estadístico.}

A través de aquello se pudo presentar y sobre todo demostrar el enunciado cualitativo de los indicadores previstos para la temática, así como la presentación de los datos recogidos en la investigación de una manera dinámica través de tablas y gráficos perfectamente comprensibles que permiten apreciar de mejor manera los resultados obtenidos en el presente trabajo de investigación para ello utilizaremos los cuadros con frecuencias y porcentajes.

\section{Población y muestra.}

\section{Identificación y caracterización de la población objetivo.}

La población está representada por 5.000 clientes que tienen un alto índice de hurto de energía y cartera vencida, los sectores del proyecto están ubicadas en zonas urbano marginal y rural del área de concesión de CNEL Regional Santo Domingo. Además, para la realización del y trabajo se contará con la participación del departamento de pérdidas de energía que está compuesto de 15 empleados, el departamento de medidores que cuanta con tres grupos de 
trabajo conformado por cuatro personas cada uno y el departamento técnico que cuenta con seis grupos de trabajo los cuales están conformados por cuatro personas cada uno

Población

58 administrativos

5000 usuarios

\section{Muestra poblacional.}

Muestra: la muestra es la que puede determinar la problemática ya que es capaz de generar los datos con los cuales se identifican las fallas dentro del proceso. El tamaño de la muestra se determina mediante la siguiente formula.

$$
n=\frac{N}{e^{2}(N-1)+1}
$$

Dónde:

$\mathrm{N}=$ tamaño de la muestra

$\mathrm{N}=$ población total

$\mathrm{E}=$ error máximo admisible $5 \%$

$\mathrm{Z}=\%$ de error $0,95 \%$

$\mathrm{P}=$ posibilidad de ocurrencia $50 \%$

$\mathrm{Q}=$ probabilidad de no ocurrencia $50 \%$

\section{Análisis de los resultados.}

Tabla 1. ¿el plan de reducción de pérdidas de energía de la empresa eléctrica cnel, de la provincia de santo domingo de los tsáchilas es eficiente?

\begin{tabular}{lcc} 
Si & $\mathbf{1 1 2}$ & $\mathbf{2 8 \%}$ \\
\hline No & 245 & $61 \%$ \\
No contesta & 44 & $11 \%$ \\
\hline
\end{tabular}

Elaborado por: grupo de investigadores

De la investigación realizada se puede establecer que, de 401 encuestados, el $28 \%$ de las personas encuestadas manifestaron que el plan de reducción de pérdidas de energía de la empresa eléctrica cnel, de la provincia de santo domingo de los tsáchilas es eficiente, 245 que representa el $61 \%$ respondieron que la gestión administrativa no es eficiente, y 44 no contestaron lo que representa el $11 \%$

Tabla 2. ¿El control que realiza la CNEL Santo Domingo para evitar las pérdidas causadas principalmente por el hurto de energía es eficiente? 


\begin{tabular}{lcl}
\hline Si & $\mathbf{9 2}$ & $\mathbf{2 3 \%}$ \\
\hline No & 297 & $74 \%$ \\
No contesta & 3 & $3 \%$ \\
\hline
\end{tabular}

Elaborado por: Grupo de Investigadores.

De la investigación realizada se puede establecer que de 401 encuestados, 92 personas que representan el $23 \%$ respondieron el control que realiza la CNEL Santo para evitar las pérdidas de energía es eficiente, 297 que representa el 74\% respondieron que la gestión financiera no es eficiente, y 3 que representa el $03 \%$ no contestaron.

Tabla 3. ¿El servicio que presta la CNEL Santo Domingo de los Tsáchilas a sus usuarios es de calidad?

\begin{tabular}{lcc}
\hline Si & $\mathbf{7 7}$ & $\mathbf{1 9 \%}$ \\
\hline No & 300 & $75 \%$ \\
No contesta & 24 & $6 \%$ \\
\hline
\end{tabular}

Elaborado por: Grupo de Investigadores.

De la investigación realizada se puede establecer que, de 401 encuestados, 77 que representan el 19\% de las personas encuestadas manifestaron el servicio brindado por el área de la salud del cantón La Concordia a sus usuarios es de calidad.300 que representa el 75\% respondieron que el servicio brindado no es de calidad, y 24 que representa el $06 \%$ no contestaron.

Tabla 4. ¿Cree Ud. ¿Que debe ejercerse control en los procesos de la CNEL para evitar las pérdidas causadas principalmente por el hurto de energía?

\begin{tabular}{lcl}
\hline Si & $\mathbf{3 3 7}$ & $\mathbf{8 4 \%}$ \\
\hline No & 48 & $12 \%$ \\
No contesta & 16 & $4 \%$
\end{tabular}

\section{Elaborado por: Grupo de Investigadores.}

De la investigación realizada se puede establecer que, de 401 encuestados, 337 que representan el $84 \%$ de las personas encuestadas manifestaron que debe ejercerse un control sobre los diferentes procesos de control para evitar robos y hurtos de energía, 48 que representa el $16 \%$ respondieron que no, y 16 que representa el $4 \%$ no contestaron.

Tabla 5. ¿Cree usted que aplicando un plan de reducción de pérdidas de energía de la empresa eléctrica CNEL de la provincia de Santo Domingo de los Tsáchilas, se reducirá el hurto de energía eléctrica por parte de sus usuarios? 


\begin{tabular}{lcc}
\hline Si & $\mathbf{3 5 3}$ & $\mathbf{8 8 \%}$ \\
\hline No & 39 & $11 \%$ \\
No contesta & 9 & $1 \%$
\end{tabular}

Elaborado por: Grupo de Investigadores.

De la investigación realizada se puede establecer que, de 401 encuestados, 353 que representan el $88 \%$ de las personas encuestadas manifestaron que aplicando un plan de reducción de pérdidas de energía de la empresa eléctrica CNEL de la provincia de Santo Domingo de los Tsáchilas, se reducirá el hurto de energía eléctrica, 39 que representa el 11\% respondieron que no, y 09 lo que representa el $01 \%$ no contestaron.

\section{Discusión.}

Mediante el estudio realizado y los datos obtenidos a través de las encuestas se puede determinar que en empresa eléctrica CNEL de la provincia de Santo Domingo-existe un deficiente e inadecuado plan para evitar las pérdidas de energía eléctrica a través del robo y hurto de sus usuarios por lo tanto es necesario desarrollar estrategias que permita incentivar una cultura de pago tendientes a la reducción de pérdidas de energía en la Corporación Nacional de Electricidad (CNEL) Santo Domingo de los Tsáchilas.

\section{Conclusiones.}

- La pérdida de energía que se producen en un sistema eléctrico tienen dos orígenes, las de carácter técnico consecuencia de la resistencia de los conductores y las impedancias de los equipos de transformación que se convierten en calor, y las de carácter comercial, debido a la lentitud e ineficiencia de los procesos administrativos, consecuencia de los errores que suelen cometerse en el proceso de lectura de los medidores, des calibración de estos errores de facturación y hurtos de electricidad por parte del usuario mediante intervenciones en el medidor o conexiones directas.

- En la corporación nacional de electricidad (CNEL) Santo Domingo de los Tsachilas, se podrían aceptar pérdidas técnicas en los sistemas de su transmisión y distribución, en el orden del $9 \%$ al $11 \%$ y las comerciales en el orden del $2 \%$ al $3 \%$ de manera que las pérdidas totales oscilen entre el $11 \%$ y $13 \%$, en una empresa con estos índices podría considerarse perdidas.

- Con las mejores propuestas para los procesos administrativos (contratación, lectura y facturación), se espera recuperar $2.450 \mathrm{Mwh}$ que representan aproximadamente $\$ 238.000$ facturados anualmente por la empresa. Cabe indicar que el estudio de mejoras y los cálculos de recuperación de energía se realizaron para la provincia de Santo Domingo específicamente. 
- La reducción de las pérdidas técnicas generalmente requieren grandes inversión es para la renovación o ampliación de las redes de distribución o de líneas de su transmisión la ampliación subestaciones etc, para lo cual las empresas deben disponer de suficientes recursos financieros y capacidad de crédito.

- Las pérdidas comerciales pueden disminuirse sustancialmente, mejorando los procesos administrativos, mediante la automatización de cada proceso y capacitando al personal de la empresa, mejorando los sistemas de facturación, mediante relativamente pequeñas inversiones en Software y Hardware, y un control sistemático de los medidores de energía, de las conexiones directas y otras prácticas de hurto de electricidad por parte de los usuarios.

\section{Referencias.}

Domingo, C. S. (2012). Manual de Funciones de Empresa Electrica S.A. Santo Domingo de los Tsáchilas.

Hernandez, \& Palafox. (2012). Administracion, Teoría, Proceso, Areas. México.

Publicaciones, C. D. (2012). Código del trabajo. Ecuador.

Plan de reducción de pérdidas de energía de la Corporación Nacional de Electricidad Regional Santo Domingo (CNEL), en la provincia de Santo Domingo de los Tsáchilas.

Jorge Luis Puyol Cortez ${ }^{5}$,Evelyn Karina Tinoco Diaz. ${ }^{6}$, Verónica Janneth Castro Celi, ${ }^{7}$, Evelyn Eugenia Alcívar Soria ${ }^{8}$ \& Efraín Velasteguí

\section{Para citar el artículo indexado.}

\footnotetext{
${ }^{5}$ Universidad Técnica Luis Vargas Torres, Facultad de Ciencias Administrativas, Santo Domingo, Ecuador, jpuyol73@gmail.com

${ }^{6}$ Universidad Técnica Luis Vargas Torres, Facultad de Ciencias Administrativas, Santo Domingo, Ecuador, kaevecris@hotmail.com

${ }^{7}$ Universidad Técnica Luis Vargas Torres, Facultad de Ciencias Administrativas, Santo Domingo, Ecuador, verito952012@hotmail.com

${ }^{8}$ Universidad Técnica Luis Vargas Torres, Facultad de Ciencias Administrativas, Santo Domingo, Ecuador, evelyn_alcivar@hotmail.com
} 
Vol. 3 / No.1/ pág. 5-15 / enero - marzo/2019

Puyo J., Tinoco E., Castro V., Alcívar E. \& Velasteguí E. (2019). Plan de reducción de pérdidas de energía de la Corporación Nacional de Electricidad Regional Santo Domingo (CNEL), en la provincia de Santo Domingo de los Tsáchilas. Revista electrónica Visionario Digital 2(2), 5-15. Recuperado desde: http://cienciadigital.org/revistacienciadigital2/index.php/VisionarioDigital/article/view/130/ 114

\section{LCiencia}

El artículo que se publica es de exclusiva responsabilidad de los autores y no necesariamente reflejan el pensamiento de la Revista Ciencia Digital.

El articulo queda en propiedad de la revista y, por tanto, su publicación parcial y/o total en otro medio tiene que ser autorizado por el director de la Revista Ciencia Digital
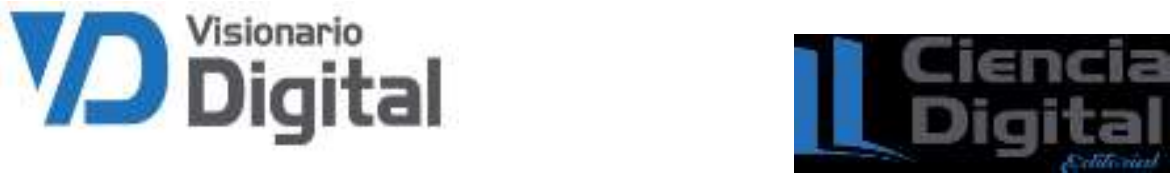